\title{
Turismo y desarrollo sustentable: contribución de Hispanoamérica
}

\author{
Rosa María Chávez Dagostino* \\ Edmundo Andrade Romo \\ Rodrigo Espinoza Sánchez \\ Universidad de Guadalajara
}

\section{Resumen}

El multicitado concepto desarrollo sustentable y su aplicación al turismo plantean la interrogante sobre la tendencia y las contribuciones del mundo académico hispanoamericano al tema. Con el fin de establecer las aportaciones y tendencias de la investigación en torno al desarrollo sustentable y el turismo, se hizo una revisión de revistas científicas en Redalyc y Eumed. Los artículos se buscaron con las palabras clave: desarrollo sustentable/sostenible y turismo. Con este criterio se seleccionaron 128 documentos, que se revisaron a partir de los enfoques débil y fuerte y sus tendencias. Se encontró que la revista con mayor contenido acerca del tema es Estudios y Perspectivas en Turismo; además, 1991 fue el año en que en Hispanoamérica se inició la discusión al respecto y el enfoque dominante fue el débil.

\section{Palabras clave}

Enfoque, sustentable/sostenible, tendencias.

Recibido: 01/07/2012 · Aceptado: 15/11/2012

*Correos electrónicos: rchavezdagostino@yahoo.com.mx · eandraderomo@gmail.com · rickylizbe@yahoo.com.mx 


\title{
Tourism and sustainable development: contribution from Hispanic America
}

\author{
Rosa María Chávez Dagostino \\ Edmundo Andrade Romo \\ Rodrigo Espinoza Sánchez \\ Universidad de Guadalajara
}

\begin{abstract}
The oft-cited concept sustainable development and its application on the field of tourism have raised the question as to the tendencies and contributions of the Hispano-American academic world on the subject. In order to establish the input and trends in the research on sustainable development and tourism, some scientific reviews considered by Redalyc and Eumed were consulted. Research was based on key words such as sustainable development, sustainability and tourism. From these criteria, a total of 128 documents were selected and reviewed considering the categories weak and strong and its trends. Estudios y Perspectivas del Turismo (Studies and Tourism Perspectives) was the review with more information on the topic; moreover, the debate on these subjects began in Hispanic America in 1991, when the dominant criterion was a weak one.
\end{abstract}

\section{KeY WORDS}

Perspective, sustainability/sustainable, trends. 


\section{Introducción}

La palabra inglesa sustainable, traducida al español como "sustentable" o "sostenible” (a veces de forma indistinta), ha sido de las más discutidas y utilizadas en los últimos 20 años de la humanidad. Su origen es contundente, nació en un proceso histórico en que se cuestionan los beneficios y la operatividad del modelo económico neoliberal. Lograr crecimiento económico sostenido (que implica permanencia) en un planeta con recursos finitos ha sido señalado como inalcanzable en numerosas ocasiones. Todos los problemas ambientales actuales: el calentamiento global, la destrucción del hábitat y la disminución de la diversidad biológica, entre otros, aunados a la pobreza, suelen relacionarse con este modelo económico. Como respuesta a este reconocimiento casi global, las acciones del mundo se han dividido en dos: mejorar el modelo actual minimizando los impactos negativos mencionados o abandonar tal modelo de desarrollo, con base en premisas económicas.

En principio, parece que el capitalismo, sistema predominante desde la caída del muro de Berlín, imagen global del finiquito del sistema socialista como opositor del capitalismo, adquirió toda la responsabilidad global, y que las contradicciones entre la economía y la ecología capitalistas generaron estos dos frentes o posturas. Sin embargo, ante una revisión más detallada del proceso que culminó con la advertencia de que se está atentando contra la propia existencia, no solo del ser humano sino de todo ser viviente, algunos autores teorizaron sobre la autodestrucción global, es decir que el planeta en su conjunto peligra verdaderamente; por ende, es importante subrayar que el capitalismo en cuanto sistema económico, desde sus primeras manifestaciones, atenta contra la naturaleza. Algo semejante ha ocurrido en los últimos años, pero ahora en el orden cultural, con los movimientos denominados "indignados", que se oponen al modelo vigente del sistema, sin negar totalmente al propio sistema.

El mundo occidental, del que Hispanoamérica es parte, conduce a la sociedad a una contradictoria necesidad de desarrollarse bajo este modelo, en general, sin un interés por cambios profundos. Frecuentemente esto se expresa también en el mundo académico.

El surgimiento de problemas ambientales globales, provocados entre otras cosas por el crecimiento demográfico y el consumo total humano superior a la capacidad de carga planetaria (Wackernagel y Rees, 1995), hizo repensar 
a la humanidad en la misma especie humana y las inequidades del desarrollo: condujo a considerar premisas de sustentabilidad. Este discurso ha causado confusión y euforia en todos los ámbitos y ha sido abordado desde diferentes disciplinas, enfoques epistemológicos y metodológicos, e incluso con perspectivas que reflejan el apego a distintas escuelas de pensamiento, en su mayoría gestadas en Occidente.

Los enfoques que se han utilizado en torno al concepto de sustentabilidad han sido muy variados, y aunque todos derivaron de la propuesta de una interacción compleja de objetivos sociales, económicos y ambientales (tres pilares de la sustentabilidad) que tienen el mismo peso (línea base triple), no han sido interpretados de modo homogéneo, por lo que las posturas de los escritos y pensadores han podido agruparse entre dos extremos limitantes: débil y fuerte (Wearing y Neil, 1999), y se pueden resumir como sigue:

1. Sustentabilidad débil: da importancia al crecimiento económico, si toma en cuenta lo ambiental es solo para mantener un stock de recursos necesarios para conseguir ese crecimiento.

2. Sustentabilidad fuerte: apela al principio precautorio, a la planeación proactiva y al monitoreo sistemático del ambiente (capital natural), por lo que se basa en los aspectos ambientales.

Según Hunter (1997), el concepto general de desarrollo sustentable evolucionó al margen del debate en curso sobre su significado. Tal aislamiento ha dado lugar a la aparición de un paradigma simplista e inflexible de turismo sostenible, que no tiene en cuenta las circunstancias específicas. Sugiere que el concepto de turismo sostenible se redefina en términos de un paradigma que incorpore una amplia gama de enfoques para el sistema turístico/medio ambiente dentro de las zonas de destino. Estos enfoques se destacan por una gran variedad de situaciones abstractas, con el objetivo de demostrar la legitimidad de las diferentes percepciones de turismo sustentable. Estableció que es posible una subdivisión entre valores extremos de los enfoques débil y fuerte. Para este estudio se asumen las siguientes definiciones construidas a partir de Hunter (1997).

- Muy débil: enfoque antropocéntrico basado en el uso óptimo de los recursos naturales; propone como viable la sustitución infinita del capital 
natural por capitales hechos por el hombre, y dirigida al continuo crecimiento económico y bienestar, cuya base se centra en la innovación tecnológica. Establece que es necesario el deterioro ambiental para lograr el crecimiento económico.

- Débil: también es un enfoque antropocéntrico que propone conservar los recursos; se preocupa por la distribución de los costos y beneficios del desarrollo buscando la equidad intra- e intergeneracional. Aunque rechaza la visión de sustitución de capital natural, reconoce puntos críticos en la agenda ambiental de conservación global y promueve que se limiten los efectos ambientales negativos del crecimiento económico.

- Fuerte: desde la perspectiva ecosistémica, identifica el valor primario de los recursos naturales y la necesidad de preservar la integridad funcional de los ecosistemas por encima del valor secundario humano; prioriza el bienestar colectivo sobre el de los consumidores, se adhiere a los principios de equidad intra- e intergeneracional y propone detener el crecimiento poblacional y económico.

- Muy fuerte: basado en la bioética, el ecocentrismo y la teoría de Gaia, donde todos los seres vivos tienen los mismos derechos de existencia, propone la preservación de los recursos naturales hasta el extremo de minimizar su uso, aun a costa de la población humana.

La popularidad del concepto de desarrollo sustentable alcanzó a casi todas las naciones y ámbitos, sus aplicaciones al turismo no son la excepción, pero en el ámbito académico, ¿cuál es la tendencia y las aportaciones de Hispanoamérica al tema de desarrollo sustentable? Con el fin de establecerlas, se hizo una revisión que incluyó a la Red de Revistas Científicas de América Latina y el Caribe, España y Portugal (Redalyc), ${ }^{1}$ y a una revista académica electrónica (TURyDES) promovida por la Red Académica Iberoamericana Local-Global, editada y mantenida por el Grupo de Investigación Eumed. ${ }^{2}$ En conjunto abarcan un total de 547 revistas y 137563 artículos. La revisión se realizó en dos pasos: la búsqueda de artículos utilizando las palabras clave: desarrollo sustentable y

${ }^{1}$ Disponible en <www.redalyc.com >

${ }^{2}$ Disponible en $<$ www-eumed.com $>$ 
turismo/desarrollo sostenible y turismo, y luego, a partir de título, palabras clave y resumen, con la condicionante de incluir en alguno de estos las tres palabras: desarrollo sustentable/sostenible y turismo. La base de datos Redalyc, en el área de ciencias sociales, a través de su hemeroteca-Sistema de Información Científica, arrojó una primera selección de 1547 artículos. De estos, se revisaron los primeros 119 documentos hasta el $2 \%$ de relevancia de acuerdo con los criterios establecidos en la búsqueda, se descartaron los trabajos realizados por autores no hispanoamericanos y quedó un total de 109. De TURyDES se revisaron los once números publicados hasta el 2011 y se seleccionaron 19 artículos con los mismos criterios. En total se revisaron 128 documentos.

Cada documento fue analizado en una base de datos conforme al tipo de documento, autores, revista, enfoque utilizado y aportaciones, información que se agrupó en dos categorías de tiempo para su análisis y comparación: $1990-2000$ y 2001-2011.

\section{Resulltados}

Alrededor de $1.13 \%$ de los artículos y $4.22 \%$ de las revistas de las bases de datos analizadas en las áreas de ciencias sociales incluyen temas donde el turismo, el desarrollo y lo sustentable/sostenible simultáneamente son palabras clave.

Se identificaron 23 revistas (cuadro 1) de publicación periódica, sobre todo con formato de artículo (gráfica 1), que han abordado los temas referidos en el periodo 1990-2011. Las primeras contribuciones hispanoamericanas al tema datan de 1991. Las revistas que han aportado mayor número de artículos relacionados con el tema por parte de autores hispanoamericanos fueron: Estudios y Perspectivas en Turismo (25.6 \%), El Periplo Sustentable (16.3 \%), TURyDES (14.7 \%), Teoría y Praxis (8.5 \%), Aportes y Transferencias (6.2 \%) y Cuadernos de Turismo (5.4\%) (cuadro 1).

Es importante resaltar que solo la revista Estudios y Perspectivas en Turismo (antes Revista Latinoamericana de Turismo) hizo publicaciones relacionadas con la temática analizada desde 1991, las demás revistas presentan aportaciones a partir del año 2000, lo que permite ubicar el año 1991 como el inicio de la discusión a través de publicaciones periódicas hispanoamericanas.

El tipo de publicación dominante en el análisis fue el artículo, bajo los requerimientos de cada revista (gráfica 1). 
CuAdro 1. Número de publicaciones revisadas por revista de las bases de Redalyc y Eumed

\begin{tabular}{lrlr}
\multicolumn{1}{c}{ Revista } & Núm. & \multicolumn{1}{c}{ Revista } & Núm. \\
Aportes y Transferencias & 8 & Industrial Data & 1 \\
Argumentos & 1 & Investigaciones Geográficas & 2 \\
Ciencia en su PC & 1 & Pasos & 5 \\
Cuadernos & 1 & El Periplo Sustentable & 21 \\
Cuadernos de Desarrollo & 1 & Quivera & 1 \\
Cuadernos de Turismo & 7 & Ra Ximhai & 1 \\
Economía y Sociedad & 1 & Revista Latinoamericana de & 3 \\
& & Turismo & 1 \\
Economía, Sociedad y Terri- & 1 & Sociedad Hoy & 11 \\
torio & & & 1 \\
Estudios Sociales & 1 & Teoría y Praxis \\
Estudios y Perspectivas en & 33 & Tropical and Subtropical \\
Turismo & & Agroecosystems & 19 \\
Gaceta Ecológica & 1 & TURyDES & \\
Gestión Turística & 6 & & \\
\hline
\end{tabular}

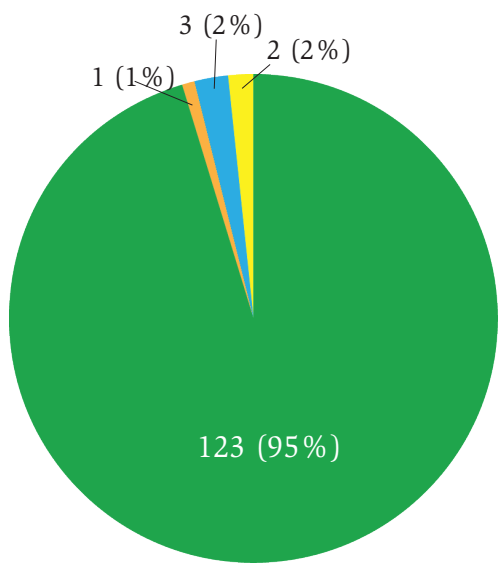

Artículo

Reseña

Ensayo

Documento especial 
Respecto al número de publicaciones que se hicieron en las revistas analizadas, puede mencionarse que a partir de 2004 se han publicado al menos seis documentos por año por parte de autores hispanoamericanos en torno al tema en cuestión. Solamente un año no se registró ninguna publicación (1992), y el año de mayor número de aportaciones fue 2010 (gráfica 2). Existe una tendencia general de crecimiento importante en el tema. En el primer periodo (1990-2000) se publicó 20.3 \% y en el siguiente (2001-2011) 79.7 \% del total analizado.

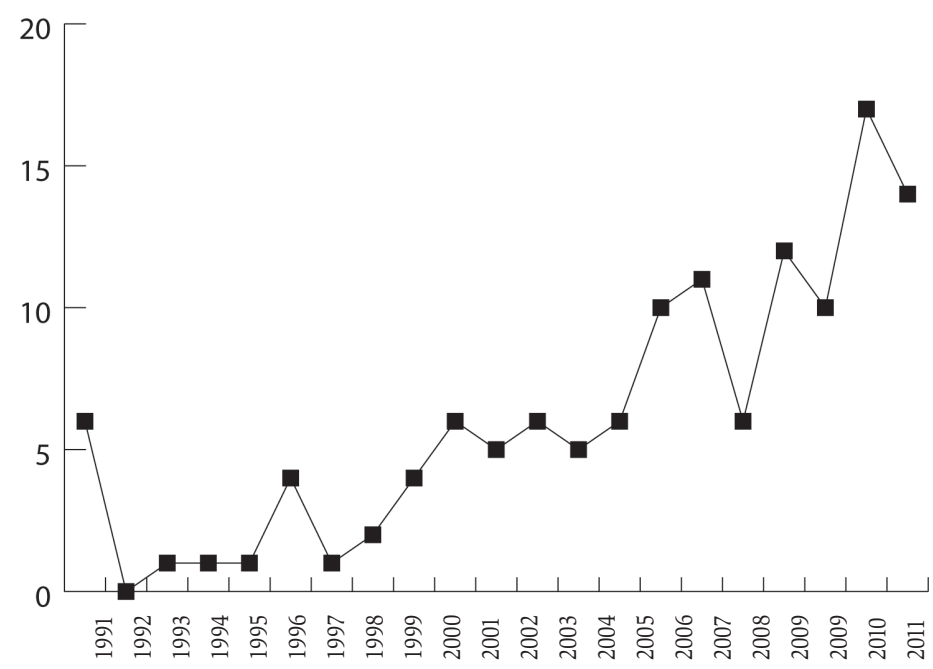

GRÁFICA 2. Número de publicaciones de autores hispanoamericanos por año en las revistas analizadas de las bases de Redalyc y Eumed de 1991 a 2011

Las palabras clave generalmente permiten identificar el eje de las aportaciones de los autores y están asociadas a un resumen, en la investigación realizada se encontró que una gran proporción de las publicaciones revisadas no las incluyen (18\%), y que su número es variable entre tres y seis, aunque muchas de ellas son en realidad "frases clave". Puede establecerse entonces, a través de estas palabras, el sentido de las aportaciones principales: turismo, desarrollo sustentable, desarrollo sostenible, ecoturismo, desarrollo y desarrollo local. Cabe subrayar que estas palabras fueron clave también para seleccionar las publicaciones en las bases de datos, sin embargo, debe resaltarse su asociación 
frecuente con las palabras desarrollo local y ecoturismo. También se encontró que las palabras sustentable y sostenible se utilizaron como palabras clave en una proporción de 2:1.

El análisis de la autoría de las publicaciones arrojó que $40.3 \%$ de los artículos fueron escritos por dos o más autores, donde al menos el primer autor fue de instituciones hispanoamericanas, sin embargo, la forma más frecuente de publicación fue la individual (59.7 \%).

En general, por su frecuencia como primer autor, sobresalen: Alfredo Ascanio, con cuatro participaciones; con tres, Manuel González Herrera, Rosa M. Chávez-Dagostino, Graciela Cruz Jiménez, Rodrigo Espinoza Sánchez, Juan C. Mantero y Rocío Serrano Barquín; con dos participaciones se registraron 11 casos como primeros autores.

\section{Enfoques y tendencias: análisis por etapas}

Periodo 1990-2000. En este periodo se localizaron 23 autores y 25 publicaciones que contienen las palabras desarrollo sustentable/sostenible y turismo en el título, resumen o palabras clave (Albornoz, 1993; Arnaiz, 1994; Ascanio, 1994; Ascanio-Lara, 1999; Boullón, 1996; Brenner, 1999; César, 1996 y 1998; Coppin, 1991; Cruz, G., 2000a y 2000b; Gurría, 1996; Juan y García, 2000; Kibedi, 1991; Mantero, 1991; Meléndez, 1999; Pérez, A., 1999; Ricaurte, 2000; Riley, 1995; Salinas-Chávez, 1998; Salinas-Chávez y Borrego, 1997; Schlüter y Gil, 1991; Serrano, 2000a y 2000b; Williams, 1991). La temática abordada va de la escala regional: América Latina y Caribe especialmente, a la nacional, donde sobresalen México, Ecuador, Venezuela y Cuba; el nivel de comunidad o localidad está poco representado. Los escritos que implican reflexiones y análisis donde se abordan temas como desarrollo, retos, perspectivas, proyecciones, planeación y diagnósticos del turismo constituyen cerca de 53 \% de la producción en este periodo. Hay una marcada orientación a los aspectos ambientales, ecológicos y el ecoturismo, ya que cerca de $30 \%$ de los trabajos en este lapso lo aborda como forma de mejorar la actividad turística o disminuir el deterioro ambiental, y es el principal justificante para insertarse en el desarrollo sustentable.

El enfoque valorado a partir de los textos, que dominó en general en esta etapa, es el fuerte, ya que cerca de 34 \% se consideró así, sin embargo, no 
con todas las características mencionadas antes (Hunter, 1997; Wearing y Neil, 1999): se identifica el valor primario de los recursos naturales, así como de los ecosistemas, su integridad y la necesidad de protegerlos, pero no el valor de lo humano, empero, prioriza el interés del bienestar colectivo mas no sobre el de los consumidores, se adhiere a los principios de equidad intra- e intergeneracional. La forma general planteada para alcanzar el desarrollo turístico sustentable es a través del monitoreo y la reducción de impactos ambientales, educación para turistas, pobladores locales y operadores turísticos, el desarrollo del turismo alternativo y la planeación. La propuesta de desarrollo local de forma explícita es casi nula.

En esta etapa sobresale el ecoturismo como una alternativa de desarrollo para Latinoamérica y se examinan los nuevos desafíos del turismo en el nivel global con propuestas de planeación, entre otras. Aunque se maneja la propuesta de una interacción compleja de objetivos sociales, económicos y ambientales para el desarrollo sustentable, predomina la preocupación ambiental; en consecuencia, la visión se fragmenta.

Periodo 2001-2011. En este periodo se produjeron 103 publicaciones que coincidieron con la búsqueda: Acerenza, 2006; Álvarez y Espinosa, 2008; Aparicio, 2004; Arnaiz y César, 2004; Ascanio, 2004, 2006 y 2007; Benseny, 2006; Berroterán y González Marcano, 2010; Boullón, 2006; Brida, Monterubbianesi y Zapata, 2011; Campodónico Pérez, 2008; Carballo, 2002 y 2005; Cardoso, 2006; Carrillo, 2010; Castro, 2011; Castro y López, 2010; César, 1996 y 1998; ChávezDagostino et al., 2006a, 2006b y 2008; Cruz, A., 2009; Cruz, G., 2000a y 2000b; Díaz Juárez et al., 2008; Duana, Rodríguez y Mota, 2011; Espinoza, ChávezDagostino y Andrade, 2007; Espinoza et al., 2010 y 2011; Flores, 2001; Fonseca, 2009; Fuller, 2011; Gallardo y Bolívar, 2010; Gómez-Nieves, 2008; González, 2004, 2007 y 2008; Hernández, 2002; Hernández y Waldo, 2002; Huerta García y Sánchez, 2011; Ibáñez, 2010a y 2010b; Inostroza, 2008; Korstanzje, 2010; León, 2007; Loperena, 2004; López Bonilla y López Bonilla , 2008; López y Palomino, 2008; López y Torres, 2010; Luciano-Toledo, Álvarez-Valdés y Castroman, 2002; Madrigal, 2002; Mantero, 2004 y 2005; Marioni y Otero, 2003; Martínez-Moreno, Ruiz Andrade y Valladares Icedo, 2009; Mendoza Ontiveros, Monterrubio Cordero y Fernández Aldecua, 2011; Mercado y Palmerín, 2009; Morales, 2011; Muñoz Piña, Rivera Planter y Oliveras Pasquel, 2005; Muñoz, 2006; Norrild, 2011; 
Osorio, J., 2003; Osorio, M., 2005; Otero, 2007; Palafox Muñoz, 2005; Palafox Muñoz y Anaya Ortiz, 2007; Pérez Serrano et al., 2010; Pérez, Cruz y Camacho, 2010; Ramírez de la 0, 2005; Ramos, 2009; Rascón, 2010; Ricaurte, 2001; Rodrigues Gomes, Castellanos Pallerols y Hernández Rodríguez, 2009; Rodríguez Herrera y Pulido Fernández, 2010; Rodríguez, 2001; Rogel Fajardo, Rojas López y Ortega Vega, 2008-2010; Rojas Hernández y Hansen Rojas, 2006; Rojas Pinilla, 2009; Rojas, 2005; Romano, 2002; Rubio Maldonado, Murad Robles y Rovira Sanroque, 2010; Salciccia, 2001; Salido Araiza et al., 2010; Salinas-Chávez y Echarri-Chávez, 2005; Salinas-Chávez y La O Osorio, 2006; Salleras, 2011; Sánchez y González, 2011; Serrano Barquín, 2008; Silveira, Silveira y Castellanos, 2010; Solari y Pérez, 2005; Sullana y Ayuso, 2003; Tarlombani da Silveira, 2005; Tiffin, Torres y Neira, 2008; Tinoco, 2003; Toselli, 2009; Trejos, 2009; Vargas, Castillo y Zizumbo, 2011; Vargas y Díaz, 2010; Vargas y Alfonso, 2011; Vázquez Solís et al., 2010; Velarde, Maldonado-Alcudia y Maldonado-Alcudia, 2009; Venturini, 2003, y Zingoni y Martínez, 2006.

En esta etapa, $43.6 \%$ de los trabajos correspondió a estudios de caso que van desde lo local, regional o estatal (63\%), hasta lo nacional (37\%).

Estas investigaciones reflejan el interés de los autores por temas como el ecoturismo, que sigue siendo muy importante en esta etapa, al igual que en la anterior. También sobresale el desarrollo de "modelos" de gestión turística con diferentes enfoques: desarrollo municipal y comunitario, autogestión basada en la comunidad, modelos de desarrollo y de análisis. El desarrollo local y endógeno, poco mencionado en la etapa anterior, adquiere mayor relevancia como un mecanismo para lograr lo sustentable, e incluso más allá del concepto de sustentabilidad que permite librarse de las prácticas neoliberales.

En cuanto a la dimensión ambiental, la conservación, el patrimonio cultural y el natural son elementos clave para la consecución de la sustentabilidad. Se reconoce el valor de las redes sociales como forma de apoyo al turismo y al desarrollo local sustentable, donde el turismo comunitario tiene un papel preponderante.

Otros temas abordados con frecuencia son el turismo alternativo, el turismo rural, los impactos del turismo en general y, en particular, del turismo de naturaleza; el papel de la planeación estratégica en el logro de los objetivos del turismo, el desarrollo y la sustentabilidad. Finalmente, los indicadores y su uso 
en las actividades/destinos turísticos son un tema recurrente que se explica por la necesidad de medir o valorar el avance en materia de desarrollo sustentable, respecto a variables ambientales, socioculturales y económicas.

Conceptos como paisaje, visión territorial, globalización, políticas públicas y la calidad de vida, ausentes en la etapa anterior, se revaloran con relación al desarrollo, al turismo y a la sustentabilidad, y adquieren importancia en esta etapa.

Las preguntas que los autores se hicieron en muchos de los casos y que guiaron sus trabajos son similares, relacionadas con el concepto de desarrollo sustentable y sus aplicaciones al turismo: ¿Discurso o acción? ¿Real o ideal? ¿Oportunidad o necesidad?

Respecto a los enfoques utilizados, aunque muchas veces no explícitos, se consideró que $13.5 \%$ de los textos abordan diversos temas en turismo bajo el enfoque débil de la sustentabilidad, en tanto que $33 \%$ utiliza el enfoque fuerte. De los trabajos en este periodo, 28 \% concuerda con que el desarrollo sustentable implica una interacción compleja de objetivos sociales, económicos y ambientales que el turismo debe abordar para contribuir en el logro de los objetivos del desarrollo sustentable, sin embargo, se pierde de vista que esta posición está limitada al turismo en sí mismo.

Reflexiones en torno a las contribuciones al conocimiento en turismo, desarrollo y sustentabilidad por parte de Hispanoamérica

En ambas etapas hay críticas profundas a los sistemas económicos que dictan las reglas y contextualizan el turismo; declaraciones y aportaciones que explican cómo en las condiciones prevalentes no podrá alcanzarse un desarrollo sustentable en sentido amplio, pero que no aportan las buscadas soluciones. Parece que la premisa es seguir haciendo lo mismo sin tener que cambiar mucho.

La sustentabilidad, al ser un proyecto de crecimiento económico más que de desarrollo humano, tiene al Estado como su principal obstáculo. La solución se encuentra en la gestión impulsada por las comunidades, donde se promueven las competencias "ecológicas" para conservar los recursos naturales, que a su vez servirán de sustento al turismo, base de la diversificación.

El Estado, al ser moderador de los efectos del crecimiento económico sobre el comportamiento humano y su entorno, tiene varias disyuntivas, según Cruz (2006): reforma la Constitución o pospone tales cambios; permite el libre flujo de capitales o lo regula; maximiza el desempleo y el subempleo o los abate; 
coadyuva a disminuir el empobrecimiento o lo extiende a la clase media; tolera la extinción de los recursos naturales o la retarda. Esto porque debe promover un comportamiento conservador de la estructura de poder tanto económica como social y política. Latinoamérica, al tomar como base al Estado con estas características, permitió la sobreexplotación de sus recursos, el desempleo, el subempleo y la gran brecha entre ricos y pobres, la deuda y la inflación. La actividad turística en Hispanoamérica en general refleja este contexto.

Tendencias emergentes e innovación en el campo de la investigación sobre el turismo sustentable a nivel mundial

Recientemente, Bramwell y Bernard (2012) analizaron, como parte de la celebración de los 20 años de existencia del Journal of Sustainable Tourism (JsT), las contribuciones internacionales sobre el tema enfocado a la innovación, el progreso y los problemas en la investigación sobre turismo sustentable. Se exponen a continuación algunas de sus conclusiones para compararlas con las contribuciones hispanoamericanas

JST publicó cerca de 500 artículos, reseñas y reportes sobre el tema en los últimos 20 años. Considera que el término turismo sustentable tomó vida en respuesta a muchos asuntos que el turismo había iniciado a cuestionar antes, desde el daño ambiental hasta los serios impactos socioculturales. En cierto grado, se reconoció que el turismo sustentable era un término relacionado con el "miedo al cambio". Aun cuando el concepto conservación era una fuerza rectora en la ideología académica en las décadas de los sesenta y ochenta, es y era en cierta medida un término que denota tanto persecución como impulso por el temor de la pérdida y de cambio. El turismo masivo y todas sus implicaciones, como el rápido crecimiento, fueron condenados por ser agentes de cambio, por ir acompañados de la explotación económica de la gente y de los lugares.

Los mismos autores explican que, poco a poco, el desarrollo del turismo se vio como una vía potencial de cambios positivos a través de las ideas de un turismo sustentable. El conocimiento del turismo sustentable ha crecido y ha establecido formas potenciales de fomentar la mejora de los resultados. El turismo sustentable es cada vez más innovador en la identificación de las formas de asegurar los beneficios positivos, además de los criterios establecidos de regulación y control del desarrollo. 
Una de las preguntas recientes se relaciona con la innovación: el turismo sustentable como concepto innovador y la innovación en la investigación sobre el turismo sustentable, considerando que la innovación requiere tres elementos: creatividad, aproximación a la resolución de problemas y una nueva forma de pensar.

Sin duda, la investigación del turismo sustentable ha estado involucrada en la resolución de problemas y ha desencadenado nuevas formas de pensar, pero es muy difícil de determinar en qué medida este tipo de investigación ha sido eficaz para influir en el cambio del mundo real. Hay muchos casos de aplicaciones reales y sustanciales de las ideas de la investigación del turismo sustentable al sector, aunque el impacto de esta investigación también es difícil de establecer.

En el mundo real del turismo se ha visto un reconocimiento cada vez mayor de la triple línea de base como un concepto, y de la noción de responsabilidad social corporativa, no obstante, ninguna de estas innovaciones ha surgido en el seno de la actividad turística o de la investigación en el turismo. Por el contrario, reflejan un pensamiento social y político más amplio, incluyen las fuerzas del mercado y la influencia de la gobernanza, así como sus interacciones. Asimismo, se ha evidenciado la necesidad de reconocer y adaptarse a los problemas del cambio climático, pero, de nuevo, esta innovación no es en absoluto exclusiva del turismo ni de sus investigadores. La innovación consistente en ampliar la participación pública y el empoderamiento local en el desarrollo turístico es muy general, pero han existido algunos elementos específicos relativos a innovaciones del turismo sustentable: manejo de visitantes, nuevas formas de alojamiento y hospedaje, turismo asociado a la conservación y a las áreas naturales protegidas, y el llamado slow tourism. Los hallazgos de Bramwell y Bernard (2012) respecto a la mayoría de las innovaciones que pueden asociarse al turismo sustentable son altamente dependientes y afectadas por procesos sociales y económicos más amplios.

En este punto de la reflexión cabe preguntarse entonces qué elementos son necesarios para la innovación en el turismo y la investigación en turismo sustentable. Aunque la innovación se considera compleja y dependiente de la escala temporal y espacial y del contexto, para lograr grandes innovaciones en la sociedad estas 
tienen que ser acogidas positivamente por la sociedad; Urry (2011) menciona que deben convertirse en "asunto de moda y deseo". Como ejemplo puede citarse el logro del paso de una economía alta en carbono a una baja en carbono, donde el turismo influye activamente y la innovación ha sido muy conservadora.

Se considera que la mayoría de los procesos de innovación en este campo son los caracterizados por la mejora constante, que se basan en normas y rutinas preestablecidas con modificaciones, buscan rapidez y producción de ganancias inmediatas, se basan en el mantenimiento de tecnologías existentes de fácil adaptación al turismo, todas estas son consideradas innovaciones incrementales; las innovaciones disruptivas de "gran avance" son escasas.

La investigación acerca del turismo sustentable evoca innovaciones más radicales que rara vez son aceptadas por los diseñadores de políticas públicas en los gobiernos, ideas y formas de gestión en el turismo tales como degrowth, steady sate tourism y slow tourism.

Algunas áreas de reciente crecimiento en el campo de la investigación sobre el turismo sustentable que son tendencias innovadoras emergentes son de carácter interdisciplinario, por ejemplo, el tema del cambio climático -relacionado con la modificación de actitudes- requiere conceptos y metodologías innovadoras derivadas de la psicología social y de la mercadotecnia. También ha habido un incremento de los temas relativos a la gobernanza del turismo sustentable, que abre nuevas interrogantes acerca de cómo las empresas y actividades se reglamentan, se gobiernan o se dirigen. Otro tema que ha repuntado se vincula con el análisis del cambio cultural, reducción de la pobreza, inequidad social y valores e ideologías que prevalecen en la sociedad. Más estudios en turismo sustentable desafían a los sistemas dominantes de acción social y las alternativas que ofrecen el conocimiento y los modos de investigación. La hegemonía en la ideología del turismo sustentable de los norteamericanos, europeos, australianos y neozelandeses, por la cantidad de instituciones e investigadores que se dedican a ello, es ahora discutida por el incremento de las aportaciones de otras partes del mundo, como China.

Por último, permanecen descuidadas algunas áreas con potencial de innovación y de desarrollo con nuevas directrices en la investigación, una de estas 
es la planeación de escenarios. La investigación también deja de lado los procesos y factores que involucran a los sectores público, privado y a la sociedad en general, que conducen a la aplicación, o la falta de aplicación de medidas para el turismo sostenible.

Como una reflexión general, los investigadores hispanoamericanos han seguido las investigaciones internacionales con contribuciones en el mismo sentido, con gran cantidad de estudios de caso que conciernen a Hispanoamérica, donde es necesaria la innovación y el trabajo interdisciplinario.

\section{Bibliografía analizada}

Acerenza, M. A. (2006). "Necesidad de precisar el contenido y el alcance de la política de desarrollo sostenible del turismo”. Aportes y Transferencias, 10 (1), 11-21.

Albornoz, J. (1993). "Situación actual del ecoturismo en Ecuador”. Estudios y Perspectivas en Turismo, 2 (4).

Álvarez R. y Y. Espinosa (2008). “Turismo y desarrollo local. Proyecto para convertir 'Cocodrilo' en pueblo turístico sostenible en un área protegida de la Isla de la Juventud (Cuba)”. Cuadernos de Turismo, 22, 9-23.

Aparicio, A. (2004). "El turismo rural: una de las alternativas al desarrollo rural en la serranía de cuenca”. Cuadernos de Turismo, 13, 51-72.

Arnaiz, S. (1996). "Desarrollo turístico y medio ambiente en el Caribe continental occidental”. Estudios y Perspectivas en Turismo, 5 (2), 147-163.

------ y A. César (2004). "Sustentabilidad, pobreza y turismo ioportunidad o necesidad?" Estudios y Perspectivas en Turismo, 13 (1-2), 160-173.

Ascanio, A. (1994). "El turismo y los impactos ambientales”. Estudios y Perspectivas en Turismo, 3 (4), 325-335.

------- (2004). "Turismo y desarrollo de la comunidad: un primer paso para rescatar la identidad cultural”. Pasos, Revista de Turismo y Patrimonio Cultural, 2 (2), 155-161.

------ (2006). "La globalización del turismo y la concentración de su riqueza”. Pasos, Revista de Turismo y Patrimonio Cultural, 4 (2), 271-277. (2007). "La globalización del turismo y la concentración de su riqueza 
en el año de 1990". Pasos, Revista de Turismo y Patrimonio Cultural, 5 (3), 277-285.

Ascanio-Lara, M. (1999). “Una aproximación al ecoturismo en Venezuela”. Estudios y Perspectivas en Turismo, 8 (3-4), 274-290.

Benseny, G. (2006). "El espacio turístico litoral”. Aportes y Transferencias, 10 (2), 102-122.

Berroterán, M. A. y Y. González Marcano (2010). “Valoración económica del paisaje para la gestión sostenible del área de playa Puerto Viejo, municipio Gómez, estado Nueva Esparta. Venezuela”. Gestión Turística, 13, 63-91.

Boullón, R. (1996). "Reflexiones sobre turismo sostenible”. Estudios y Perspectivas en Turismo, 5 (1).

------- (2006). "Espacio turístico y desarrollo sustentable”. Aportes y Transferencias, 10 (2), 17-24.

Brenner, L. (1999). "Modelo para la evaluación de la 'sustentabilidad' del turismo en México con base en el ejemplo de Ixtapa-Zihuatanejo”. Investigaciones Geográficas (Mx), 39, 139-158.

Brida, J., P. Monterubbianesi y S. Zapata (2011). "Impactos del turismo sobre el crecimiento económico y el desarrollo. El caso de los principales destinos turísticos de Colombia”. Pasos, Revista de Turismo y Patrimonio Cultural, 9 (2), 291-303.

Campodónico Pérez, R. A. (2008). "El turismo y los vaivenes del desarrollo". Aportes y Transferencias, 12 (1), 15-40.

Carballo, A. (2002). "Participación comunitaria en el desarrollo del ecoturismo sustentable en el Caribe mexicano". Estudios y Perspectivas en Turismo, 11 (3-4), 300-310.

(2005). "Análisis de un modelo de desarrollo ecoturístico en Quintana Roo". Teoría y Praxis, 1, 31-47.

Cardoso, C. (2006). “Turismo sostenible: una revisión conceptual aplicada”. El Periplo Sustentable, 11, 5-21.

Carrillo, S. T. (2010). "La contabilidad medioambiental: perspectivas de su desarrollo en el sector turístico cubano”. TURyDES, 3 (8), 1-18.

Castro, D. A. (2011). "Turismo instrumento o panacea de desarrollo municipal en Colombia”. TURyDES, 4 (9), 1-4.

Castro, U. y J. H. López (2010). “Desarrollo regional y turismo: revisión histórica estructural de la Riviera Nayarit, México”. TURyDES, 3 (8), 66-96. 
César, A. (1996). "Desarrollo sustentable, turismo y medio ambiente en el Caribe”. Estudios y Perspectivas en Turismo, 5 (1), 18-49.

(1998). "Ecosistemas costeros y desarrollo turístico". Estudios y Perspectivas en Turismo, 7 (1-2).

Chávez Dagostino, R. M., et al. (2006a). "Huella ecológica y turismo sustentable”. Teoría y Praxis, 2, 147-156.

Chávez Dagostino, R. M., E. Andrade Romo y R. Espinoza Sánchez (2006b). "Patrimonio, turismo y desarrollo sustentable". Teoría y Praxis, 2, 9-23.

Chávez Dagostino, R. M., et al. (2008). "Huellas ecológicas y sustentabilidad en la costa norte de Jalisco, México”. Teoría y Praxis, 5, 137-144.

Coppin, L. (1991). "Ecoturismo y América Latina: Una aproximación al tema”. Estudios y Perspectivas en Turismo, 1 (1), 3-10.

Cruz, A. (2009). "Factores de atracción turística: similitudes y diferencias en los principales destinos del Caribe Insular”. TURyDES, 2 (6), 1-18.

Cruz, G. (2000a). "Ecoturismo y turismo sustentable”. El Periplo Sustentable, 1. (2000b). "Protocolos o avances de investigación organización comunitaria y turismo sustentable en San Miguel Almaya, México”. El Periplo Sustentable, 2.

Díaz Juárez, I., et al. (2008). "Potencial para turismo alternativo del municipio de paso de ovejas, Veracruz". Tropical and Subtropical Agroecosystems, 8 (2), 199-208.

Duana, D., J. S. Rodríguez y C. E. Mota (2011). “Ecoturismo y su impacto en el desarrollo regional en Hidalgo (2010)”. TURyDES, 4 (10), 19-41.

Espinoza, R., R. M. Chávez Dagostino y E. Andrade (2007). “Análisis patrimonial de la región baja del río Tomatlán”. TURyDES, 1 (1), 9-18.

Espinoza, R., et al. (2010). “Un ‘trinomio perfecto’ basado en: turismo, desarrollo sustentable y calidad de vida para el desarrollo comunitario de Chacala Nayarit, México". TURyDES, 3 (8), 51-65.

Espinoza, R., et al. (2011). "Breve estudio sobre la oferta de las empresas de turismo de naturaleza en el municipio de Cabo Corrientes, Jalisco, México". TURyDES, 2 (4), 1-9.

Flores, E. (2001). “Ecoturismo y turismo sustentable”. El Periplo Sustentable, 3. Fonseca, M. (2009). "Punta Mita en la dinámica del desarrollo turístico regional”. El Periplo Sustentable, 16, 85-108. 
Fuller, N. (2011). "Reflexiones sobre el turismo rural como vía de desarrollo". Estudios y Perspectivas en Turismo, 20 (4), 929-942.

Gallardo, G. y D. Bolívar (2010). "USIACURI: modelo de autogestión comunitaria en el desarrollo turístico y ambiental en el Departamento del Atlántico-República de Colombia”. TURyDES, 3 (7), 1-11.

Gómez-Nieves, S. (2008). “Ciencia y desarrollo turístico en México”. Estudios y Perspectivas en Turismo, 17 (3-4), 340-358.

González, M. (2004). “Gestión ambiental para un modelo sostenible”. Estudios y Perspectivas en Turismo, 13 (1-2), 35-49.

------- (2007). "Política turística para el desarrollo sostenible del turismo cultu-

ral / San Juan de los remedios Cuba”. Teoría y Praxis, 3, 53-63.

------- (2008). "Puesta en valor turístico sustentable de la Amazona peruana”. Teoría y Praxis, 5, 247-267.

Gurría Di-Bella, M. (1996). “Turismo moderno de orientación ecológica”. Estudios y Perspectivas en Turismo, 5 (4), 325-340.

Hernández Llosas, M. (2002). "Patrimonio cultural y desarrollo sostenible en la quebrada de Humahuaca potencial y perspectivas”. Cuadernos de Turismo, 18, 153-158.

Hernández, L. y L. Waldo (2002). "La sustentabilidad en la Marquesa como alternativa de desarrollo turístico". El Periplo Sustentable, 6.

Huerta García, M. y Á. Sánchez (2011). “Evaluación del potencial ecoturístico en áreas naturales protegidas del municipio de Santa María Huatulco, México”. Cuadernos de Turismo, 27, 521-539.

Ibáñez, R. (2010a). "Sustentabilidad e indicadores de desarrollo turístico en México”. TURyDES, 3 (7), 12-34.

------- (2010b). “Crecimiento económico, desarrollo sustentable y turismo: Una aproximación del posicionamiento de Baja California Sur (BCs) en el Barómetro de Sustentabilidad”. El Periplo Sustentable, 20, 75-118.

Inostroza, V. (2008). "Aportes para un modelo de gestión sostenible del turismo comunitario en la región andina”. Gestión Turística, 10, 77-90.

Juan, G. y S. García (2000). “Turismo y sustentabilidad”. El Periplo Sustentable, 2. Kibedi, G. (1991). “Turismo, ecología y ecoturismo”. Revista Latinoamericana de Turismo, 1 (2), 130-131. 
Korstanzje, M. (2010). “Turismo y desarrollo: la construcción del lujo y el ocio en el mundo contemporáneo”. TURyDES, 3 (7), 12-26.

León, M. E. (2007). “Turismo sostenible y biodiversidad en la Orinoquia Colombiana”. TURyDES, 1 (1), 1-8.

Loperena, Á. (2004). “Turismo y desarrollo sostenible”. El Periplo Sustentable, 9. López Bonilla, J. y L. López Bonilla (2008). "La capacidad de carga turística”. El Periplo Sustentable, 15, 123-150.

López, G. y B. Palomino (2008). "Políticas públicas y ecoturismo en comunidades indígenas de México”. Teoría y Praxis, 5, 33-50.

López, M. y C. Torres (2010). "Redes sociales en proyectos ecoturísticos”. Teoría y Praxis, 7, 101-114.

Luciano-Toledo, G., J. Álvarez-Valdés y A. Castroman (2002). "Planificación estratégica de empresas de turismo y desarrollo sustentable del sector: Propuesta de un modelo". Estudios y Perspectivas en Turismo, 11 (3-4). Madrigal, D. (2002). "Los problemas coyunturales de la planeación en México y sus impactos en la sustentabilidad y el ordenamiento territorial”. El Periplo Sustentable, 5.

Mantero, J. (1991). "Reflexiones sobre la planeación turística y el desarrollo de la comunidad”. Revista Latinoamericana de Turismo, 4.

(2004). "Desarrollo local y actividad turística”. Aportes y Transferencias, 8 (1), 11-38.

------ (2005). "Del turismo del territorio interior al turismo de nodalidades turísticas”. Aportes y Transferencias, 9 (2), 167-185.

Marioni, S. y A. Otero (2003). "Desarrollo turístico sustentable”. Estudios y Perspectivas en Turismo, 12 (1-2), 169-180.

Martínez-Moreno, O. C., J. C. Ruiz Andrade y O. L. Valladares Icedo (2009). "Las particularidades de la agenda 21 para el turismo mexicano. Un análisis de la aplicación del sistema de indicadores de Sustentabilidad en el municipio de Playas de Rosarito, B.C. México”. Gestión Turística, $12,9-29$.

Meléndez, A. (1991). "Desarrollo turístico en América Latina”. Revista Latinoamericana de Turismo, 1 (3), 188-207.

(1999). "Estrategias para el desarrollo sostenible del turismo en América

Latina”. Estudios y Perspectivas en Turismo, 8 (1-2). 
Mendoza Ontiveros, M. M., J. C. Monterrubio Cordero y M. J. Fernández Aldecua (2011). "Impactos sociales del turismo en el centro integralmente planeado (CIP)”. Gestión Turística, 15, 47-73.

Mercado, H. y Palmerín, M. (2009). "El turismo como fuente de desarrollo del estado de Michoacán”. TURyDES, 2 (5), 1-19.

Morales, S. (2011). "Turismo en espacios rurales de Perú”. Estudios y Perspectivas en Turismo, 20 (3).

Muñoz Piña, C., M. Rivera Planter y C. Oliveras Pasquel (2005). “Turismo y conciencia ambiental en México”. Gaceta Ecológica, 75, 5-18.

Muñoz, J. (2006). “Aproximación panorámica a la Carta europea del turismo sostenible en espacios protegidos". Estudios y Perspectivas en Turismo, 15 (3), 236-254.

Norrild, A. (2011). “De la inversión hotelera al turismo sustentable”. Estudios y Perspectivas en Turismo, 20 (4).

Osorio, J. (2003). "Hacia una agenda del desarrollo sustentable en Chile”. El Periplo Sustentable, 8.

Osorio, M. (2005). "El entorno ambiental y el turismo sustentable, un análisis social”. El Periplo Sustentable, 10.

Otero, A. (2007). "La importancia de la visión de territorio para la construcción de desarrollo competitivo de los destinos turísticos”. Cuadernos de Turismo, 19, 91-104.

Palafox Muñoz, A. (2005). “Turismo sustentable”. Teoría y Praxis, 1, 97-107.

y J. S. Anaya Ortiz (2007). "Reflexiones en torno a la implementación de la agenda 21 en Cozumel”. Gestión Turística, 7, 103-128.

Pérez Serrano, A. M., et al. (2010). "Turismo rural y empleo rural no agrícola en la Sierra Nororiente del estado de Puebla: caso red de Turismo Alternativo Totaltikpak, A. C”. Investigaciones Geográficas (Mx), 71, 57-71.

Pérez, A. (1999). "Impactos turísticos". Estudios y Perspectivas en Turismo, 8 (1-2).

Pérez, C., G. Cruz y A. Camacho (2010). “Análisis del aprovechamiento turístico del parque estatal Sierra de Tepozotlán, México desde la perspectiva de la sustentabilidad”. El Periplo Sustentable, 19, 35-68.

Ramírez de la O, I. (2005). "Turismo alternativo en los bosques de Atlautla, Estado de México”. Ra Ximhai, 1 (3), 523-557. 
Ramos, A. L. (2009). "Propuesta de desarrollo turístico sustentable en municipios ubicados en la región de la Cañada en el Estado de Oaxaca 2008”. TURyDES, 2 (5), 20-54.

Rascón, E. (2010). "La ruta de tránsito y su vinculación con el desarrollo del turismo en Panamá: el pasado y el presente”. TURyDES, 3 (8), 37-50.

Ricaurte, C. (2000). "Desarrollo sustentable, gestión local y turismo”. El Periplo Sustentable, 1.

(2001). "Desarrollo turístico sustentable: el caso de Ixtapan de la Sal”. El Periplo Sustentable, 3.

Riley, M. (1995). "Análisis del desarrollo del turismo en las Islas Malvinas". Estudios y Perspectivas en Turismo, 4 (2).

Rodrigues Gomes, H. A., G. Castellanos Pallerols y N. Hernández Rodríguez (2009). "Modelo integrado para la factibilidad de inversiones turísticas en zonas costeras". Ciencia en su PC, 4, 3-15.

Rodríguez Herrera, I. M., J. I. Pulido Fernández (2010). “Factores condicionantes de la sostenibilidad como una dimensión estratégica del desarrollo turístico mexicano". Cuadernos de Turismo, 25, 125-146.

Rodríguez, B. (2001). "Programa de desarrollo sustentable en la región de la Mariposa Monarca”. El Periplo Sustentable, 4.

Rogel Fajardo, I., A. Rojas López y S. Y. Ortega Vega (2008-2010). "El turismo alternativo como estrategia de conservación de la Reserva de la Biósfera de la Mariposa Monarca”. Quivera, 13 (2), 115-133.

Rojas Hernández, J. y G. Hansen Rojas (2006). “Turismo de naturaleza, desarrollo local sustentable y megaproyectos hidroeléctricos en la Patagonia chilena”. Sociedad Hoy, 11, 87-108.

Rojas Pinilla, H. (2009). "Entre lo ideal y lo real; ¿los cambios en los enfoques propuestos de turismo rural sostenible desde la Organización de las Naciones Unidas contribuirían al desarrollo rural territorial?" Cuadernos de Desarrollo Rural, 6 (62), 145-171.

Rojas, J. (2005). "Implicaciones del turismo en la ecología y el desarrollo de la amazonia en Napo, Ecuador”. Teoría y Praxis, 1, 49-67.

Romano, Segrado (2001). "El desarrollo sustentable y turismo de Ocuilan”. El Periplo Sustentable, 4.

(2002). "La sustentabilidad como alternativa social”. El Periplo Sustentable, 6 . 
Rubio Maldonado, E., M. Murad Robles y J. V. Rovira Sanroque (2010). “Crisis ambiental en la costa de Quintana Roo como consecuencia de una visión limitada de lo que representa el desarrollo sustentable”. Argumentos, 23 (63), 161-185.

Salciccia, D. (2001). "El ecoturismo rural y el desarrollo sustentable del patrimonio natural e histórico cultural”. Estudios y Perspectivas en Turismo, $10(1-2)$.

Salido Araiza, P. L., et al. (2010). "El patrimonio natural y cultural como base para estrategias de turismo sustentable en la Sonora Rural”. Estudios Sociales, 17 (especial), 80-103.

Salinas-Chávez, E. (1998). “Turismo en Cuba: Desarrollo, retos y perspectivas”. Estudios y Perspectivas en Turismo, 7 (1-2).

Salinas-Chávez, E. y O. Borrego (1997). “Consideraciones sobre el desarrollo del ecoturismo en Cuba”. Estudios y Perspectivas en Turismo, 6 (3-4).

Salinas-Chávez, E. y M. Echarri-Chávez (2005). “Turismo y desarrollo sostenible: el caso del centro histórico de la Habana, Cuba”. Pasos, Revista de Turismo y Patrimonio Cultural, 3 (1).

Salinas-Chávez, E. y J. La O Osorio (2006). "Turismo y sustentabilidad: De la teoría a la práctica”. Cuadernos de Turismo, 17, 201-221.

Salleras, L. (2011). "Territorio, turismo y desarrollo sustentable en la quebrada de Humahuaca”. Estudios y Perspectivas en Turismo, 20 (5).

Sánchez, L. y R. González (2011). “Destinos turísticos de montaña con migración de amenidad”. Estudios y Perspectivas en Turismo, 20 (2).

Schlüter, R. y G. Gil (1991). "El turismo en el mundo: su proyección al siglo xxı". Estudios y Perspectivas en Turismo, 1 (2).

Serrano Barquín, R. C. (2008). "Hacia un modelo teórico-metodológico para el análisis del desarrollo, la sostenibilidad y el turismo”. Economía, Sociedad y Territorio, 8 (26), 313-355.

Serrano, R. (2000a). "Fundamentación para la planeación del turismo sustentable, hacia el desarrollo local”. El Periplo Sustentable, 2. (2000b). "Protocolos o avances de investigación en el sur del Valle de Toluca una alternativa de desarrollo sustentable”. El Periplo Sustentable, 1.

Silveira, Y., R. Silveira y G. Castellanos (2010). “Impacto social y económico de la industria turística cubana”. TURyDES, 3 (8), 19-36. 
Solari, A. y M. Pérez (2005). "Desarrollo local y turismo: relaciones, desavenencias y enfoques”. Economía y Sociedad, X (16), 49-64.

Sullana, P. y S. Ayuso (2003). “Desarrollo sustentable”. Estudios y Perspectivas en Turismo, 12 (3-4).

Tarlombani da Silveira, M. A. (2005). "Turismo y sustentabilidad. Entre el discurso y la acción”. Estudios y Perspectivas en Turismo, 14 (3).

Tiffin S., X. Torres y F. Neira (2008). "Actividades ecoturísticas y clusters en Chile”. Estudios y Perspectivas en Turismo, 17 (3-4).

Tinoco, O. (2003). "Los impactos del turismo en el Perú”. Industrial Data, 6 (1), 47-60.

Toselli, C. (2009). "Programa de fortalecimiento a destinos turísticos emergentes: Un análisis desde la visión del desarrollo endógeno”. Gestión Turística, 12, 109-124.

Trejos, B. (2009). "Redes de apoyo al turismo comunitario en Costa Rica". TURyDES, 2 (6), 19-59.

Vargas, E., M. Castillo y L. Zizumbo (2011). “Turismo y sustentabilidad”. Estudios y Perspectivas en Turismo, 20 (3).

Vargas, T. y M. Díaz (2010). "Metodología para el diagnóstico de la capacitación en el turismo sostenible con enfoque de organización que aprende". TURyDES, 3 (7), 23-45.

Vargas, T. y D. Alfonso (2011). "Modelo sistémico de gestión de la capacitación para el turismo sostenible en Viñales Cuba”. TURyDES, 4 (9), 1-15.

Vázquez Solís, V., et al. (2010). "Evaluación de los atractivos naturales para el desarrollo del ecoturismo en la región huasteca de San Luis Potosí, México”. Cuadernos de Turismo, 25, 229-245.

Velarde, M., A. Maldonado-Alcudia y M. Maldonado-Alcudia (2009). "Pueblos mágicos: estrategia para el desarrollo turístico sustentable”. Teoría y Praxis, 6, 79-93.

Venturini, E. J. (2003). "Patrimonio cultural, turismo y desarrollo local: el camino de las estancias jesuíticas de Córdoba”. Aportes y Transferencias, 1, 45-64.

Williams, P. (1991). "Desafío en el manejo del turismo ecológico". Estudios y Perspectivas en Turismo, 1 (2). 
Zingoni, J. M. y S. M. Martínez (2006). “La planificación estratégica como herramienta de concertación público-privada para el desarrollo turístico. El caso Patagonés”. Aportes y Transferencias, 10 (2), 83-101.

\section{Fuentes consultadas}

Bramwell, B. y L. Bernard (2012). “Towards Innovation in Sustainable Tourism Research?” Journal of Sustainable Tourism, 20 (1), 1-7.

Cruz, L. (2006). “El problema de la sustentabilidad”. Enfoques, XIII (1-2), 145-158.

Hunter, C. (1997). "Sustainable Tourism as an Adaptative Paradigm”. Annals of Tourism Research, 24 (4), 850-867.

Urry, J. (2011). Climate Change and Society. Cambridge: Polity Press.

Wackernagel, M. y W. Rees (1995). Our Ecological Footprint: Reducing Human Impact on the Earth. Gabriola Island: New Society Publishers.

Wearing, S. y J. Neil (1999). Ecotourism. Impacts, Potentials and Possibilities. Oxford: Butterworth-Heinemann Publishers. 